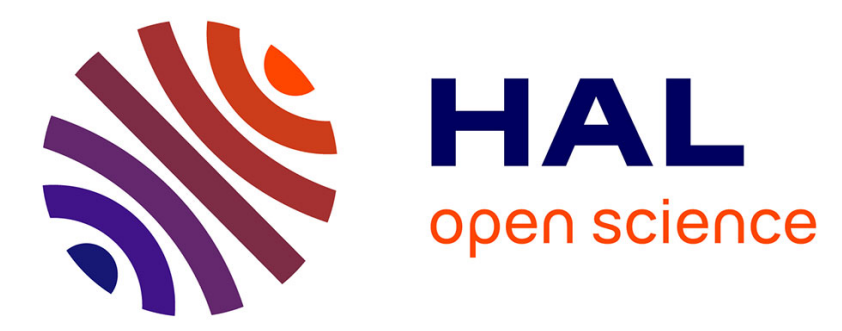

\title{
Video analysis of dust events in full-tungsten ASDEX Upgrade
}

\author{
F. Brochard, A. Shalpegin, S. Bardin, T. Lunt, V. Rohde, J.L. Briançon, G. \\ Pautasso, C. Vorpahl, R. Neu, Asdex Upgrade Team
}

\section{To cite this version:}

F. Brochard, A. Shalpegin, S. Bardin, T. Lunt, V. Rohde, et al.. Video analysis of dust events in fulltungsten ASDEX Upgrade. Nuclear Fusion, 2017, 57 (3), 10.1088/1741-4326/aa4e56 . hal-01834094

\section{HAL Id: hal-01834094 \\ https://hal.univ-lorraine.fr/hal-01834094}

Submitted on 10 Jul 2018

HAL is a multi-disciplinary open access archive for the deposit and dissemination of scientific research documents, whether they are published or not. The documents may come from teaching and research institutions in France or abroad, or from public or private research centers.
L'archive ouverte pluridisciplinaire HAL, est destinée au dépôt et à la diffusion de documents scientifiques de niveau recherche, publiés ou non, émanant des établissements d'enseignement et de recherche français ou étrangers, des laboratoires publics ou privés. 


\title{
Video analysis of dust events in full-tungsten ASDEX Upgrade
}

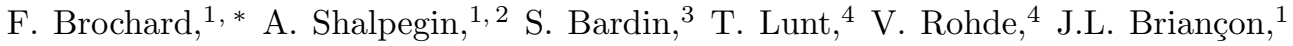 \\ G. Pautasso ${ }^{4}$ C. Vorpahl,${ }^{5}$ R. Neu ${ }^{4,6}$ and the ASDEX Upgrade Team ${ }^{4}$ \\ ${ }^{1}$ Université de Lorraine, Institut Jean Lamour, UMR 7198 CNRS, Vandoeuvre-lès-Nancy, France \\ ${ }^{2}$ Universität Stuttgart, IGVP, Pfaffenwaldring 31,70569, Stuttgart, Germany \\ ${ }^{3}$ FOM Institute DIFFER, Dutch Institute For Fundamental Energy Research, \\ Edisonbaan 14, 3439MN Nieuwegein, The Netherlands \\ ${ }^{4}$ Max Planck Institut für Plasmaphysik, Boltzmannstrasse 2, 85748 Garching, Germany \\ ${ }^{5}$ ITER Organization, F-13067, St Paul les Durance, France \\ ${ }^{6}$ Technische Universität München, Boltzmannstrasse 15, 85748 Garching, Germany
}

(Dated: November 10, 2016)

\begin{abstract}
Fast video data recorded during seven consecutive operation campaigns (2008-2012) in fulltungsten ASDEX Upgrade have been analyzed with an algorithm developped to automatically detect and track dust particles. A total of 2425 discharges have been analyzed, corresponding to 12204 seconds of plasma operation. The analysis aimed at precisely identifying and sorting the discharge conditions responsible of the dust generation or remobilization. Dust rates are found to be significantly lower than in tokamaks with carbon PFCs. Significant dust events occur mostly during off-normal plasma phases such as disruptions and particularly those preceded by vertical displacement events (VDEs). Dust rates are also increased but to a lower extent during type-I ELMy H-modes. The influences of disruption energy, heating scenario, vessel venting and vessel vibrations are also presented.
\end{abstract}

\section{INTRODUCTION}

Despite important breakthroughs have been achieved in the last decades in the understanding of fusion plasmas, there are still numerous problems to be solved before the realization of a secure and sustainable fusion energy source. One of the major issues is a detailed comprehension of the plasma-wall interaction mechanisms and their consequences on the device performance and safety [1, 2]. In fusion devices, plasma facing components (PFCs) are exposed to high particle fluxes, large heat loads and neutron irradiation and have to endure relatively high thermal and mechanical constraints. These processes damage and erode the PFCs and limit their lifetime, making it sometimes necessary to procede to maintenance shutdowns to replace the damaged components. The erosion of PFCs also leads to the formation of dust, which can potentially cause several operational concerns: for instance, dust migration in the vessel and the related release of impurities to the hot core plasma is responsible for high core radiation, which reduces the performance of fusion plasmas and may cause disruption, particularly in long pulse discharges [3]; dust accumulation may also degrade mirrors and diagnostics, or lead to erroneous temperature measurements of the PFCs. The DITS campaign on Tore Supra suggests that dust may be an operational limit if a fixed plasma scenario is used repeatedly [4, 5. Dust accumulation can also lead to more serious safety issues, such as tritium retention or explosion in case of air ingress or contact with water steam.

Although the operation of present day tokamaks such as AUG only seldomly suffer from dust, these issues are

\footnotetext{
* frederic.brochard@univ-lorraine.fr
}

expected to be more problematic in the future ITER tokamak due to longer discharge durations, larger energy and power deposition on PFCs and operation with D-T plasmas. The understanding of dust production rates, localization, remobilization and transport in the vacuum vessel during plasma phases in present tokamaks, particularly those with metallic walls, is therefore of primary importance for improving the reliability of dust models and predictions in the perspective of ITER and future fusion reactors.

Among the various diagnostics used for in situ investigations of dust in tokamaks (laser light scattering [6], dust collection by aerogels [7, dust detectors [8, 9], standard CCD cameras [10], etc...), fast imaging is one of the most valuable for investigating dust behaviour during plasma operation. Compared to other diagnostics, which probe localized areas, fast cameras have the ability to probe a significant plasma volume (typically $\sim 20 \%$ of the torus volume per camera in AUG) while keeping relatively high temporal resolution. Their main drawback is that only the brightest dust particles can be detected, i.e. those with sizes of the order of a few microns or more, heated at a sufficient temperature [11. Post-mortem analyses suggest that only a small fraction of dust particles produced in AUG fit these conditions 12 14. However these large dust particles are those which might have the strongest impact on tokamak performance and are thus of particular interest. A second drawback of fast imaging is the huge amount of data produced which requires sophisticated analysis procedures. An algorithm, named TRACE (Tracking and Classification of pinpoint Events), has been specifically developped in order to automatically detect and track dust particles in plasmas directly from fast camera data [15]. TRACE has been adapted to tokamaks in order to efficiently anal- 
yse video data on a day-to-day basis, making it possible to carry out a statistical analysis of dust events in ASDEX Upgrade under various discharge conditions [16.

This paper is organized as follows. The fast imaging diagnostic used to record dust events during AUG discharges as well as the main features of the TRACE algorithm are presented in section II. A survey of dust in disruptive discharges is presented in section III. Section IV focuses on the influence of type-I ELMs, heating sources, vessel opening and vessel vibrations on dust observation rates in non-disruptive discharges. Main limitations of the analysis are discussed in section V. Finally section VI concludes the paper and gives an outlook on future investigations.

\section{DATA ACQUISITION AND VIDEO PROCESSING}

\section{A. Description of measurements}

In order to investigate the amount of dust mobilized during AUG discharges two fast framing cameras (Phantom V7.1 and Phantom V7.3) were used. Videos are typically recorded at 10,000 frames per second (fps) with $256^{*} 256$ pixels and 14 bit depth resolution resulting in 8 GB data per shot. Fast cameras have also been used for non dust-related investigations. For that reason, they have been installed at different locations around the torus and various optical filters have been used depending on the main experimental purposes. Filters, used in less than $9 \%$ of the analyzed videos, mostly aimed at attenuating plasma emission (anti-D $\alpha$ and near IR filters), highlighting impurities radiation (mostly W-I, C-II or CIII), highlighting plasma emission ( $D \alpha$ filters) or reducing saturation problems (neutral filters). The use of such filters may influence dust detection, nevertheless no clear effect could be identified in our analysis. This is possibly due to the fact that dust radiation is never totally filtered out since it results from both thermal radiation of dust grain and line radiation of dust ablation cloud [17. It also certainly demonstrates the effectiveness of the automatic detection procedure presented in section II B. The cameras locations have been changed up to 8 times in the frame of individual experimental campaign. The focal length of the camera optics has also been varied from $4 \mathrm{~mm}$ to $8 \mathrm{~mm}$, resulting in different views depicted in Fig.1. As a consequence, PFCs, heating antennas, diagnostics and other elements susceptible to plasma contact and thus likely to generate dust in the vessel, may differ according to the cameras fields of view. Therefore, despite, the plasma volume probed by the cameras varies by less than $20 \%$ in the discharges which have been analyzed in our study, noticeable differences can occur in the rates of dust observed according to the camera configuration. These differences contain information on the most favorable areas for dust formation or remobilization and are therefore investigated in our study provided enough shots have been recorded in similar conditions to ensure reliable statistics. For this reason, a particular attention is paid to two camera views highlighted in Fig.1. one imaging the divertor from the top of sector 1 (636 shots, encompassing $4.25 \mathrm{~m}^{3}$ of plasma volume) and one imaging the upper part of the vessel from the midplane in sector 8 (1482 shots, encompassing a plasma volume of $3.56 \mathrm{~m}^{3}$ ). It has also to be noted that some AUG sectors, particularly sectors 7 and 15, where neutral beam injectors are installed, were not observed with fast cameras. It means that dust mobilized or created in these areas can not be detected with the cameras, unless its lifetime and total displacement is long enough, which is very improbable [18. It is also important to underline that the minimum size of dust particles which are visible with fast cameras depends on their temperature, i.e. on the plasma parameters, as well as on their composition and on the optical arrangement [19, 20]. Despite fast framing cameras can resolve smaller and faster particles than standard rate cameras due to their higher contrast ratio, it is expected that they can detect only a small fraction of the total dust population. The deliberate injection of calibrated dust of known morphology and composition is an efficient procedure to determine this minimum size, but it has not been performed on AUG in the course of this investigation. If one refers to such dedicated experiments realized on DIII-D, TEXTOR and Pilot-PSI [2123. it seems reasonable to state that the typical dust size which can be seen by fast framing cameras in standard fusion plasmas conditions is around $5 \mu \mathrm{m}$ in diameter, and down to about $1 \mu \mathrm{m}$ in the most favorable conditions. According to the post-mortem analysis of dust collected in AUG in 2009, the dust size distribution is best fitted with truncated log-normal distribution functions, with a maximum probability around $1 \mu \mathrm{m}$ in diameter for $\mathrm{W}$ spheroids [14. If one considers, according to this work, that the diameter of $\mathrm{W}$ spheroids in AUG is correctly fitted with a log-normal distribution with geometrical mean value $\mu=0.9 \mu m$ and standard deviation $\sigma=1.9$, then $44 \%$ of collected particles have a diameter above $1 \mu \mathrm{m}$ but only $0.4 \%$ have a diameter above $5 \mu \mathrm{m}$. This low fraction actually represents a significant mass fraction of the W spheroid inventory. Indeed, by considering a distribution truncated to $35 \mu \mathrm{m}$, diameter of the largest spheroid collected, W spheroids above $1 \mu \mathrm{m}$ represent about $95 \%$ of the total mass of $\mathrm{W}$ spheroids and those with a diameter above $5 \mu \mathrm{m}$ still account for $22 \%$ of the total mass of $\mathrm{W}$ spheroids. The size distribution of conglomerates of $\mathrm{B}, \mathrm{C}$ and $\mathrm{W}$, which can be spheroids or irregularly shaped, is also best fitted with log-normal distribution with a maximum shifted to smaller scales. Considering log-normal distributions with $\mu=0.5 \mu \mathrm{m}$ and $\sigma=3.65$ for C-dominated dust and $\mu=0.3 \mu \mathrm{m}$ and $\sigma=3.51$ for other conglomerates, one gets respectively $30 \%$ and $3.6 \%$ of $\mathrm{C}$ dust and $17 \%$ and $1.2 \%$ of conglomerate dust above $1 \mu \mathrm{m}$ and $5 \mu \mathrm{m}$ in diameter. Considering a distribution truncated to $53 \mu \mathrm{m}$, equivalent circle diameter of the largest conglomerate collected, conglomerates above 

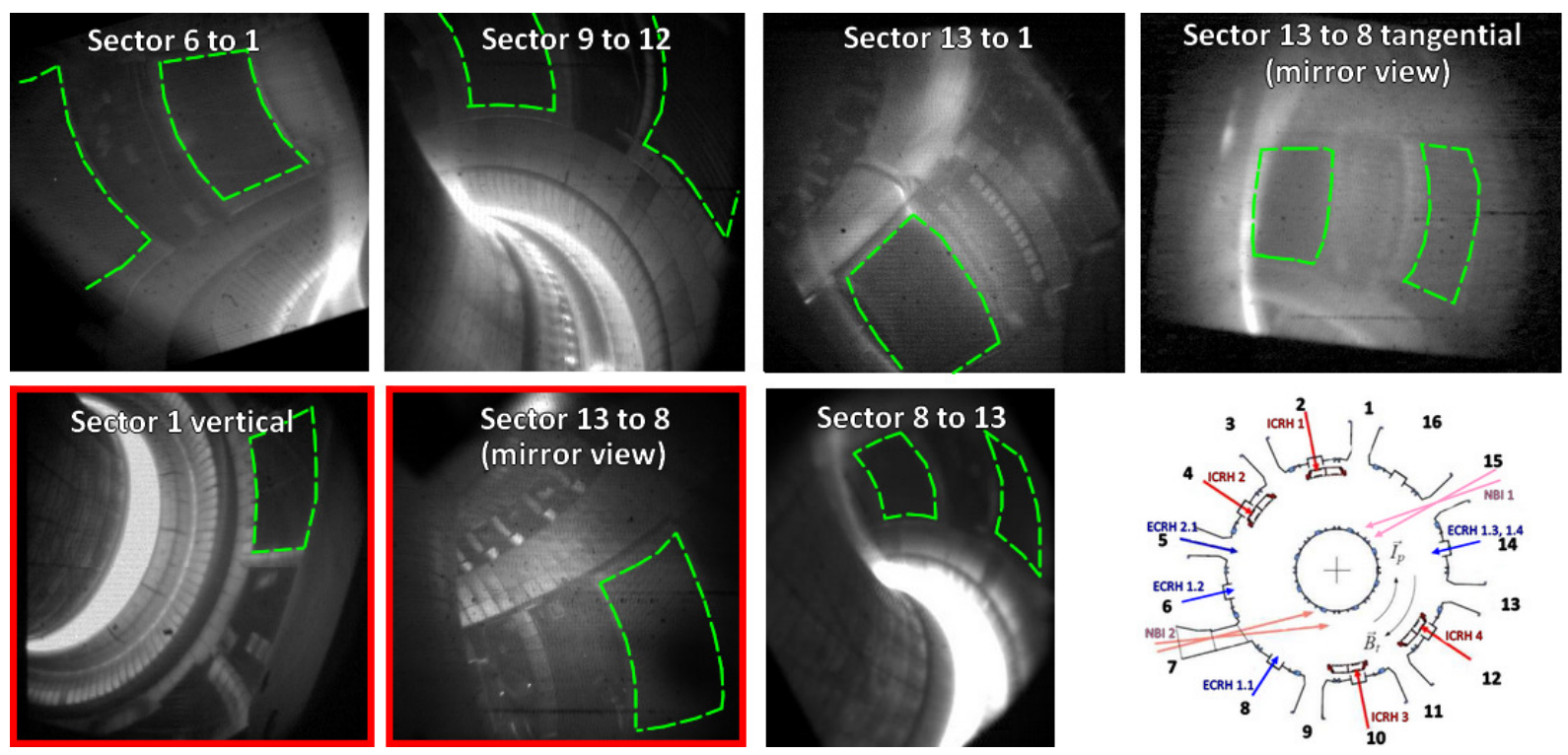

FIG. 1. Pictures showing the different fields of view of the fast cameras used for dust investigations in AUG, as well as a top view scheme of AUG figuring the 16 sectors. The contours of ICRH antennas are shown in green dashed lines. The two views highlighted by red squares depict views of particular interest discussed in the paper.

$1 \mu m$ represent about $99.5 \%$ of the total mass of conglomerates and those with a diameter above $5 \mu \mathrm{m}$ still account for $89 \%$ of the total mass of conglomerates.

On the one hand, spheroids have experienced a loss of mass which can be significant during their interaction wih the plasma [18, hence their size can be several times larger shortly after mobilization, thus facilitating their observation. On the other hand, collected irregularly shaped conglomerates, which can have comparatively large sizes, have fragile structures implying the absence of intensive contact with the plasma and it can be concluded that their radiation level is probably too low to be observable by fast visible cameras. All in all, it is therefore very likely that despite only a few percents of the intrinsic dust population mobilized in AUG can be seen with fast cameras, this population of relatively large particles may represent a significant mass fraction of the total dust inventory.

The work presented in this paper is mostly based on the statistical analysis of 3251 movies recorded in independent discharges covering five consecutive operation campaigns (i.e. discharges AUG $\sharp 23174$ to $\mathrm{AUG} \sharp 28695$, corresponding to the period 2008-2012). About 30 TB of video data have been analyzed, which corresponds to 15470 seconds of plasma operation including all kinds of discharge conditions. However, in order to ensure a better comparison between all the shots, only the movies corresponding to similar frame rates (i.e. in between 5,000 fps and 20,000 fps) and probing similar plasma volume where kept. As a consequence, the results presented in this paper focus on 2425 discharges, representing a total of 12204 seconds of plasma operation and 16591 dust particles trajectories.

\section{B. Automatic dust detection}

Video data are analyzed with TRACE, an efficient video analysis tool coded in MATLAB developped for tracking dust particles in laboratory or fusion plasmas [15, 16, 24. TRACE has become a versatile tool which has also been proved to be efficient for other detection and tracking applications [25]. In fusion plasmas, dust particles observed with fast cameras appear as bright spots, i.e. local maxima of light intensity. The detection method used in TRACE relies on the combination of several thresholds computed for each frame over three different spatial scales. For an event to be considered as a potential dust particle, its brightness has to exceed three thresholds $T h r_{G l o b}, T h r_{R e g}$ and $T h r_{L o c}$ computed as follows:

$$
\begin{gathered}
T h r_{G l o b}=\frac{1}{n} \sum_{j=1}^{n}\left(I_{j}-\frac{1}{2}\left|I_{j}-\bar{I}\right|\right) \\
T h r_{R e g}(R)=I_{\text {min }}(R)+\frac{I_{\text {max }}(R)-I_{\text {min }}(R)}{10} \\
\operatorname{Thr}_{L o c}(L)=\frac{1}{n} \sum_{j=1}^{n} I_{j} * f\left(T h r_{R e g}(R)-N_{s}\right)
\end{gathered}
$$

with $f\left(T h r_{R e g}(R)-N_{s}\right)=1.1+\frac{T h r_{R e g}(R)-N_{s}}{10} \leq 1.6$,

where the indices $j=1 \ldots n$ correspond to each pixel of a single frame and where $I_{j}$ is the intensity of pixel 
j. $T h r_{G l o b}$ is a global threshold, function of the average light intensity $\frac{1}{n} \sum_{j=1}^{n} I_{j}$ and light standard deviation $\bar{I}$ computed over the whole frame size. $T h r_{R e g}$ is called regional threshold and is a function of the maximum and minimum light intensity $I_{\max }$ and $I_{\min }$ in windows $R$ of size $32 \times 32$ pixels. $T h r_{L o c}(L)$ is a local threshold computed at each pixel as a function of the average light intensity in windows $L$ of size $9 \mathrm{x} 9$ pixels. It depends on the difference between $T h r_{R e g}$ and $N_{s}$, which is an approximated value of the thermal noise of the camera sensor. In order to determine $N_{s}$, the camera is triggered to record a few hundreds of frames before or after any light emission in the plasma vessel. $N_{s}$ is the threshold value for which no dust particle is detected in these frames. In addition, for an event to be considered as a dust particle, it has to have a local maximum of intensity in subwindows of size $5 \times 5$ pixels. This local maximum of intensity is considered as the center of coordinates of the dust particles. Such an assumption is faster than using the centroid approximation (weighted sum of light intensity) and sufficient in our case since dust trajectories are not studied.

This combination of thresholds makes it possible to detect particles even in case of strong plasma illumination like disruptions or MARFEs, under the condition that the dust is not located in light-saturated areas (i.e. areas without any local peak of light intensity). Contrary to many other detection procedures, the frame background is not substracted, making it possible to detect stationary events such as hot spots, but also slow dust particles. Indeed, depending on the displacement of dust particles with respect to the camera lines of sight, it is possible that dust particles apparently do not move for a few frames. On average, the mean displacement of dust particles between 2 frames is found to be 0.3 pixel at $10,000 \mathrm{fps}$.

The tracking procedure combines two strategies. For newly detected dust particles, the tracking is based on the minimization of the total square displacements for all possible combinations of displacements between subsequent frames, provided a maximum displacement of $M_{d}=5$ pixels per frame per particle. This means that dust particles moving faster than $M_{d}$ pixels per second are not tracked, however such particles are extremely rare (except during disruptions). Once dust particles are tracked for at least two frames, their tracking is based on the linear extrapolation of their displacement (within the same range $M_{d}$ ), i.e. the combinations providing best alignement is chosen. If no good alignement is found, the minimization of the total square displacement within $M_{d}$ is applied, making it possible to track more complex trajectories. Finally, in order to ensure that only dust fly-bys are taken into account (and not, e.g. residual noise or hot spots), only the events tracked at least 20 frames with a total displacement of at least 5 pixels are considered in this study.

\section{DUST IN DISRUPTIVE SHOTS}

\section{A. Main tendencies}

It is well known that plasma dust content is significantly larger in disruptive discharges compared to non disruptive ones [26]. Figure 2 depicts the number of shots with more than 10 dust events in the camera field of view as a function of the number of shots passed since previous disruption. It clearly evidences that most significant dust events are observed in disruptive shots, or in the very first shots after a disruption: on a total of 344 fast camera movies with more than 10 dust events, 209 correspond to disruptive shots and 40 to shots immediately preceded by a disruption. Several basic mechanisms can link dust to disruptions: i) dust may be produced by transient heat loads on the PFCs during the disruption [27, ii) dust previously deposited onto the PFCs might be remobilized during the disruption by thermal shocks, by mechanical vibrations of the PFCs or by forces due to large currents induced by fast transients [27, 28] and iii) disruption might be due to the presence of an excessive amount of dust in the plasma [3]. It is nearly impossible to say whether dust is produced or only remobilized during a given disruption when analyzing fast video data. Comparing the temporal evolution of dust released in the plasma with the time at which disruption occurs can only shed light on the causality link between dust releases and disruptions. As an example, Figure 3 shows a typical situation where disruption precedes a sudden increase of the dust concentration in the plasma. Disruption time is evidenced by the large and narrow peak in the total radiated power at $\mathrm{t}=1.45 \mathrm{~s}$. The $\mathrm{W}$ core concentration, $c_{W}$, derived from measurements of the grazing incidence spectrometer at $5 \mathrm{~nm}$, is also plotted and does not show any significant increase before the disruption occurs, staying well below the values observed earlier in the discharge. Several dust events are observed between $\mathrm{t}=1 \mathrm{~s}$ and $\mathrm{t}=1.1 \mathrm{~s}$ together with an increase in the total radiated power but are not accompanied by any significant increase of $c_{W}$. Then the total radiated power decreases while a single dust particle is observed for $0.1 \mathrm{~ms}$, about $0.1 \mathrm{~ms}$ before the disruption occurs. In such a case, the evolutions of $c_{W}$ and of the number of dust events suggest another cause for the disruption and it is possible to conclude that the most significant dust release is caused by the disruption. A very different case is illustrated by Figure 4. which is representative of a few tens of shots where a significant increase of dust is already observed before the disruption. It can be seen that this increase is almost concomitant with an increase in $c_{W}$, which comes after strong oscillations in the total radiated power. The detailed investigation of this shot reveals that a control instability leads to plasma contact with unconditioned wall regions. This contact presumably causes the injection of dust into the plasma as well as the increase in $c_{W}$. Finally, it is likely that the discharge terminates because of the control instability, possibly supported by the 
impurity and dust radiation.

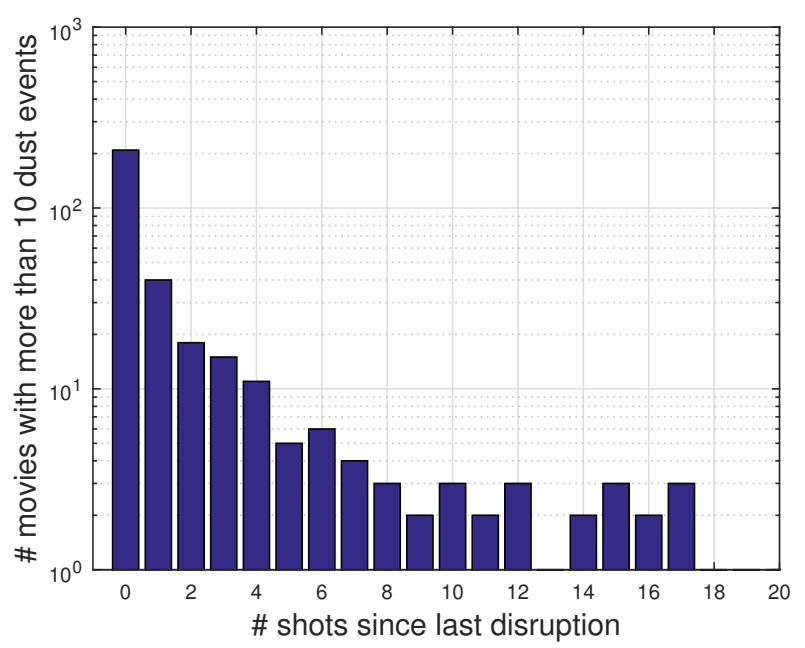

FIG. 2. Histogram of the number of fast camera movies with more than 10 dust fly-bys as a function of the number of discharges elapsed since previous disruption.

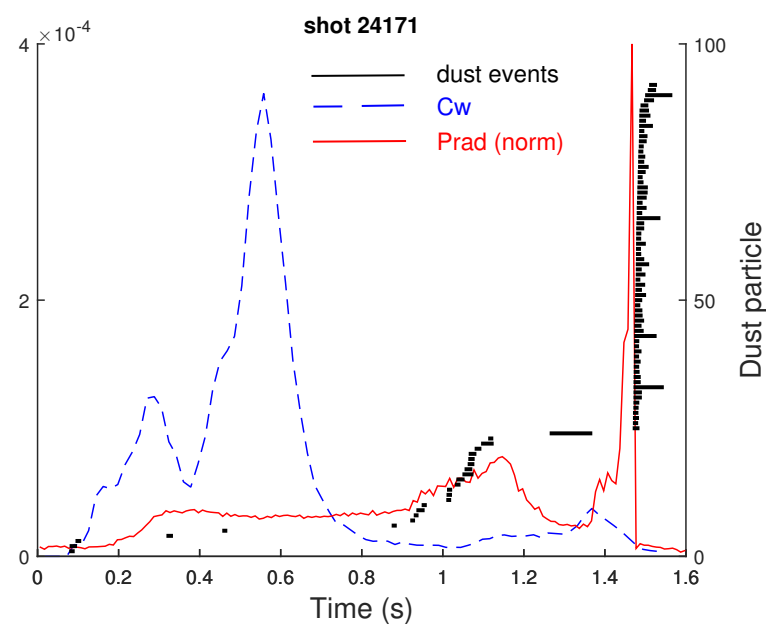

FIG. 3. W core concentration (blue dashes), total radiated power (red line) and number of dust particles detected as a function of time in disruptive shot AUG 24171. Each black horizontal dash corresponds to a single dust particule, the length of the segment giving the duration of its observation.

The statistical analysis of 479 fast camera movies of unmitigated disruptive shots shows that on average 5.3 dust particles are tracked before the disruption and 7 after the disruption. Actually, visual inspection of the movies reveals that a non negligible fraction of dust particles produced or mobilized during the disruption can not be tracked by TRACE due to their very high velocity (displacement between consecutive frames larger than 5 pixels). On addition, at the exact time of disruption, the camera data can also be fully saturated for a

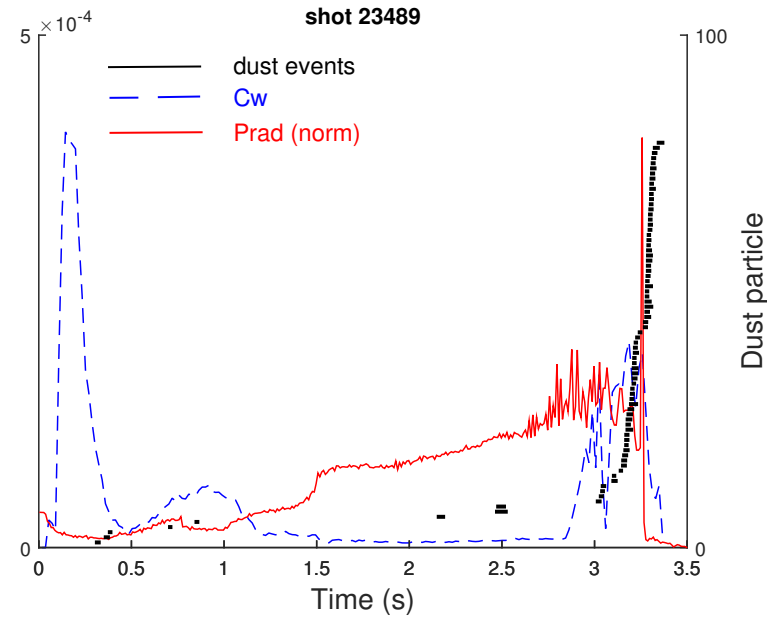

FIG. 4. W core concentration (blue dashes), total radiated power (red line) and number of dust particles detected as a function of time in disruptive shot AUG 23489.

few frames, preventing any possible detection for a short duration (typically less than $2 \mathrm{~ms}$ ). In most cases, the amount of dust released in the vessel after the disruption which can be seen with fast camera but cannot be tracked is therefore larger by a factor of a few units.

A more detailed investigation reveals some noticeable discrepancies in between consecutive experimental campaigns, as presented in Figure 5 During the first campaign, between shots $\sharp 23140$ and $\sharp 23672$, most discharges appear as dust-free or with low levels of dust before disruption occurs, but after disruption only $20 \%$ of these discharges appear as dust-free and most of them exhibit significant levels of dust. This trend is much less pronounced during the second campaign, but more surprisingly the opposite tendency is observed for the last 3 campaigns, between shots $\sharp 24190$ and $\sharp 27118$. It could be argued that some discrepancies might point out the limitation of a purely statistical approach, which mixes up various discharges scenarios and configurations and does not discriminate bias due to operational failures. For instance, a major bias can be the release of dust during the ramp-up phase of discharges immediately following another disruptive discharge. In order to filter out such a possible bias, only discharges not immediately precedeted by another disruption are considered in Figure 6 . While there is no significant difference in the dust levels after disruption, the ratio of shots which appear as free of dust before disruption is surprinsingly lower in this case and even significantly lower if one considers discharges of the two campaigns which took place between shots $\sharp 24190$ and $\sharp 25945$. All in all, dust levels in disruptive shots have significantly changed after shot $\sharp 24189$, older shots presenting the expected behaviour, i.e. dust levels larger after than before disruption, while later shots present the opposite tendency. The reason seems to be that the wall behaved differently during these campaigns 
due to different wall conditionning. Indeed, boron layers were removed by cleaning after shot 24226 and there was no boronization in between shots 24190 and 25336 and no vent in between these two campaigns (see also Figure 14 and section IV-C).

(a)

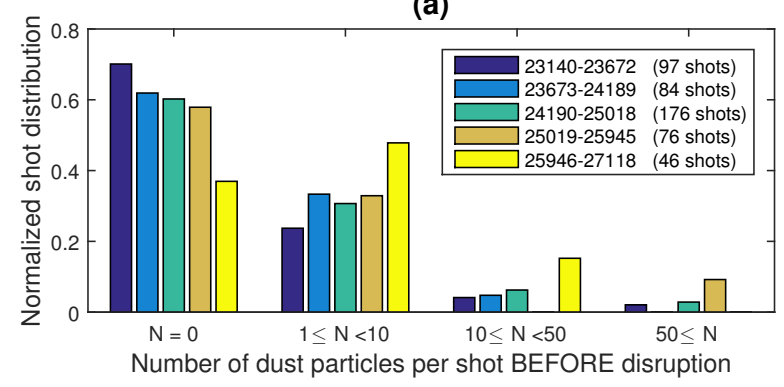

(b)

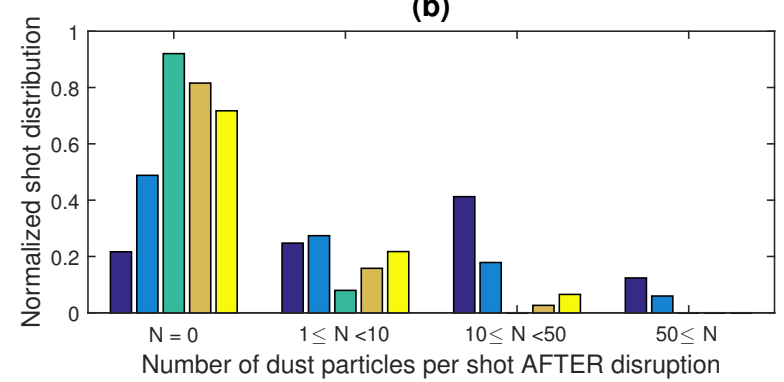

FIG. 5. Normalized distribution of shots as a function of the number of dust particles detected before disruption (top) and after disruption (bottom) in unmitigated disruptive discharges. Colors refer to five consecutives experimental campaigns. Numbers in the legend of top figure correspond to the number of dusruptive discharges analyzed per campaign.

Mixing results for the five experimental campaigns reported here reveals that there is no detection of any dust fly-by in $40 \%$ of unmitigated disruptions and in $56 \%$ of non-disruptive discharges. Of course, dust might be too small, too cold or too fast to be observed by the camera, or released in sectors of the vessel which are not covered by camera, but these results highlight the absence of thick deposited layers for W PFCs in ASDEX Upgrade in comparison to tokamaks with carbon PFCs where dust investigations with fast cameras have also been carried out 20]. No dust fly-by is seen in $59 \%$ of disruptions occuring in the current ramp-up phase (51 movies), in $33 \%$ of disruptions occuring in the flattop phase (211 movies) and in $44 \%$ of disruptions happening in the current rampdown phase (214 movies). It is therefore questionable whether dust release is proportional to the energy contained in plasma when the disruption occurs, as reported in Tore Supra 9. (a)

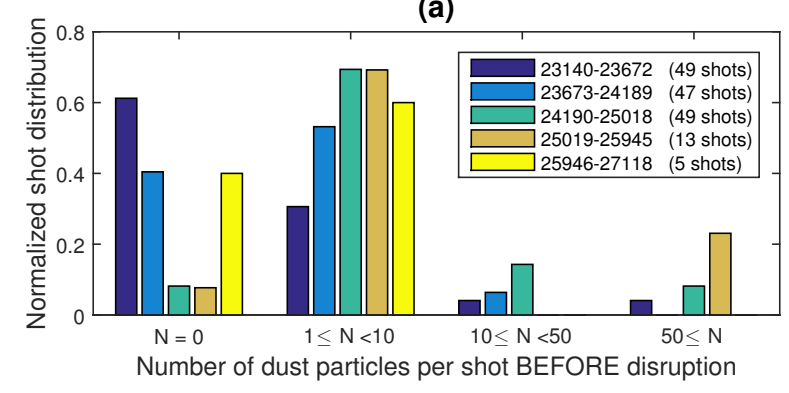

(b)

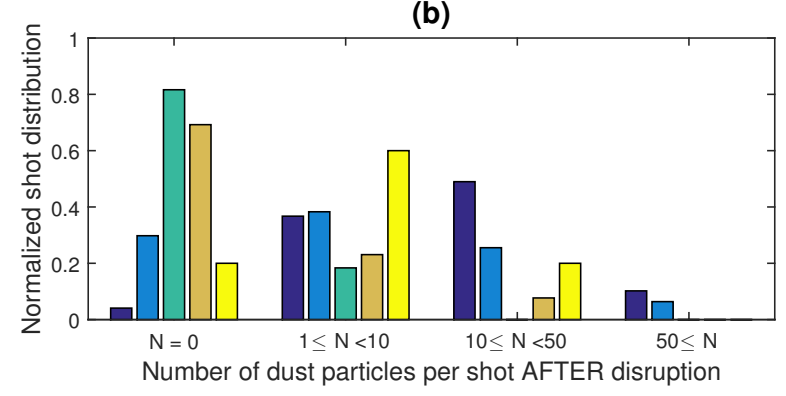

FIG. 6. Same figure as Fig. 5 but for discharges not immediately preceded by another disruptive discharge.

\section{B. Influence of disruption energy}

Figure 7 depicts the number of dust fly-bys observed after disruptions as a function of the energy contained in the plasma $5 \mathrm{~ms}$ before disruption for 479 unmitigated disruptions and 63 mitigated disruptions. Despite the rather large dispersion, one can notice that the probability to observe significant dust events first increases with the disruption energy, reaches a maximum around $10^{5} \mathrm{~J}$ and then decreases at larger energies. There is no obvious reason for a reduction of dust generation as the heat loads increase under present AUG discharge conditions (significant $\mathrm{W}$ evaporation and resulting vapor shielding would require larger heat loads [29]), but partial melting of metallic dust may take place, increasing dust adhesion to the PFCs. Therefore, the evolution depicted in Figure 7 for energies larger than $10^{5} \mathrm{~J}$ may result from a reduction in dust remobilization due to the melting of metallic dust. One can also not exclude the possibility that this maximum results from a statistical bias, since there is a relative lack of data for unmitigated disruptions at large energies: while unmitigated disruptions below $10^{5} \mathrm{~J}$ represents $81 \%$ of the analyzed shots, disruptions above $2.10^{5} \mathrm{~J}$ represents only $6 \%$.

Disruption mitigation by the use of Massive Gas Injection [30] is found to decrease the intensity of dust events. Indeed, an average value of 2.8 dust fly-bys is observed after mitigated disruption, compared to an average value of 7 dust fly-bys in the case of similar discharges with unmitigated disruption. 


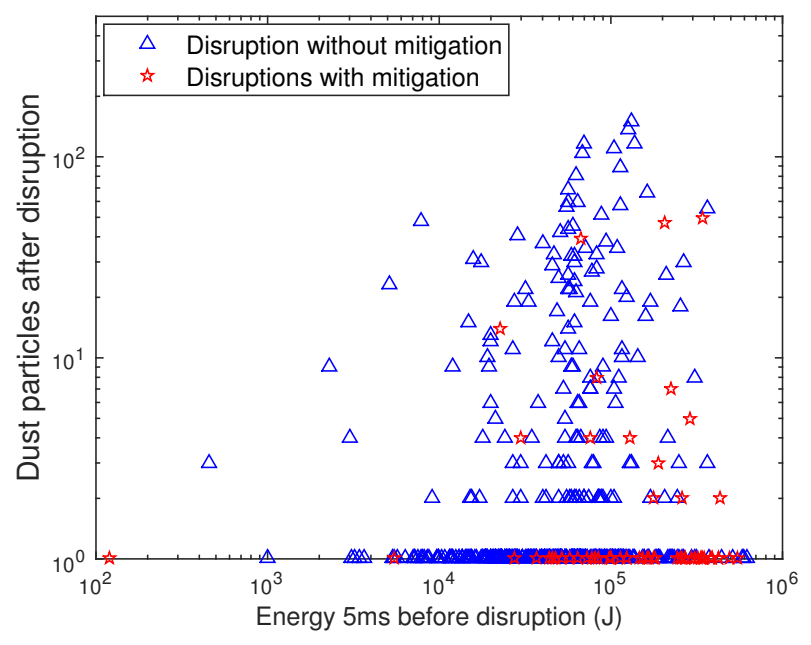

FIG. 7. Number of dust particles detected after disruptions as a function of disruption energy.

\section{Influence of plasma vertical displacements}

Vertical displacement events (VDEs) significantly increase heat fluxes to the PFCs. Our analysis demonstrates that the probability of large dust events is slightly increased in disruptions preceded by VDEs, as it can be seen in Figure 8. However, the effects are dissimilar according to the direction of the vertical displacement: while both upward and downward VDE increase the risk of large dust events before disruption, the probability of observing large dust events after disruption is lower in disruptions preceded by downward VDE compared to disruptions without VDE. Disruptive discharges with downward VDE also have the highest probability to be free of any detected dust event after disruption.

The examination of the energy contained in the plasma shortly before the disruption might explain these observations, as presented in Table I. While the maximum energy during the discharge is, on average, very similar in both downward and upward VDEs cases, the plasma energy measured shortly before the disruption is on average twice less in downward VDE disruptive discharges compared to upward VDE and no VDE cases. Hence, the lower dust rates observed after disruption in the case of downward VDE are likely to result from a lower disruption energy, which would be in concordance with Fig.7.

The amplitude of vertical displacements also impacts significantly dust observation rates: figure 9 highlights that large displacements result in larger amount of dust whatever the experimental campaign. The evaluation of individual campaigns also reveals that dust rates are divided by a factor of about 2 for the largest displacements after shot 25945 compared to older shots. The reason might be the installation of Resonant Magnetic Perturbation (RMP) coils after shot 25945. RMP was only seldomly operated in the shots investigated in our study (i.e. in 2 shots with upward VDE and in 10 shots with downward VDE), making it impossible to assess the influence of the magnetic perturbations on dust rates, but with the installation of saddle coils, PFCs have been replaced with new tiles. We assume that the absence of any deposit on these tiles explains the changes observed after shot 25945 .

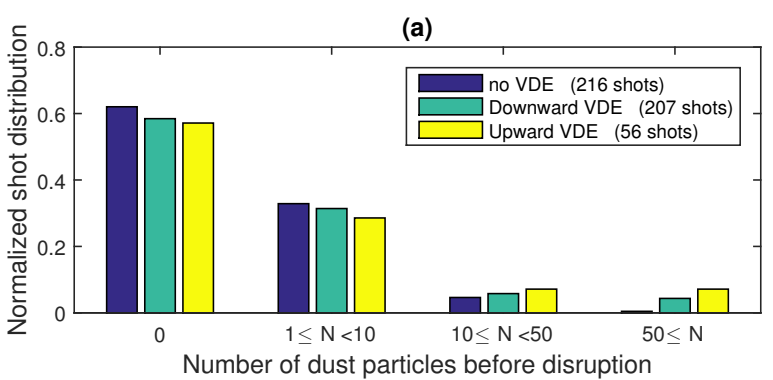

(b)

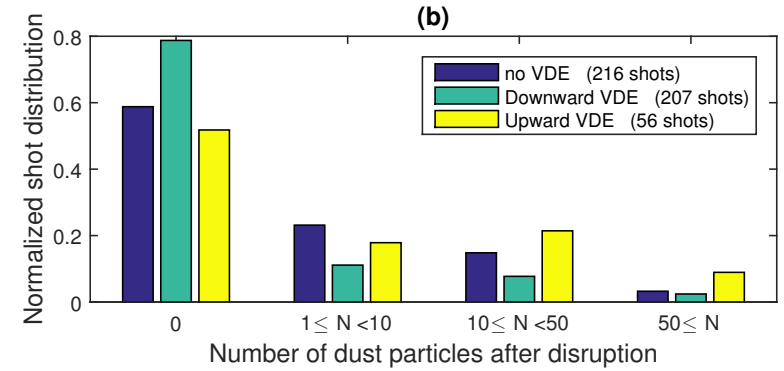

FIG. 8. Normalized distribution of shots as a function of the number of dust particles in disruptive shots without VDE (blue), in disruptions with downward VDE (green) and in disruptions with upward VDE (yellow). Top picture presents dust rates before disruption and bottom picture present dust rates after disruption.

\section{DUST IN NON DISRUPTIVE SHOTS}

\section{A. Influence of type-I ELMs}

Results presented in previous section have established the existence of a correlation between the intensity of disruptions and the amount of dust mobilized in AUG discharges. It is questionable whether other transient events such as ELMs have noticeable effects on the rates of mobilized dust as well. Theoretical studies suggest that thermal shocks caused by ELMs could efficiently remobilize dust grains in ITER [27] and correlation establishing an increase of dust production with the presence of large ELMs has been reported on DIII-D in the case of submicron dust particles observed by Rayleigh/Mie scattering [6]. The observation by fast camera of the release of dust particles resulting from ELMs interacting with PFC has also been reported in DIII-D 31, demonstrating that micron-size dust might also be remobilized by 


\begin{tabular}{|l|l|l|l|l|}
\hline & $\begin{array}{l}\text { Probability to detect } \\
\text { more than 10 dust } \\
\text { particles before/after } \\
\text { disruption }\end{array}$ & $\begin{array}{l}\text { Ratio of shots } \\
\text { without dust } \\
\text { event }\end{array}$ & $\begin{array}{l}\text { Average plasma energy } \\
5 \text { ms before disruption } \\
(\mathrm{kJ})\end{array}$ & $\begin{array}{l}\text { Average plasma maxi- energy (kJ) } \\
\text { mats }\end{array}$ \\
\hline No VDE (216 shots) & $5.1 \% / 18 \%$ & $38 \%$ & 86 & 380 \\
\hline Downward VDE (207 shots) & $10.1 \% / 10.1 \%$ & $46 \%$ & 43 & 429 \\
\hline Upward VDE (56 shots) & $14.3 \% / 30.3 \%$ & $32 \%$ & 85 & 426 \\
\hline
\end{tabular}

TABLE I. Influence of VDEs on dust rates, as well as on the plasma energy $5 \mathrm{~ms}$ before disruption. Maximum plasma energy during the discharge is also given for comparison.

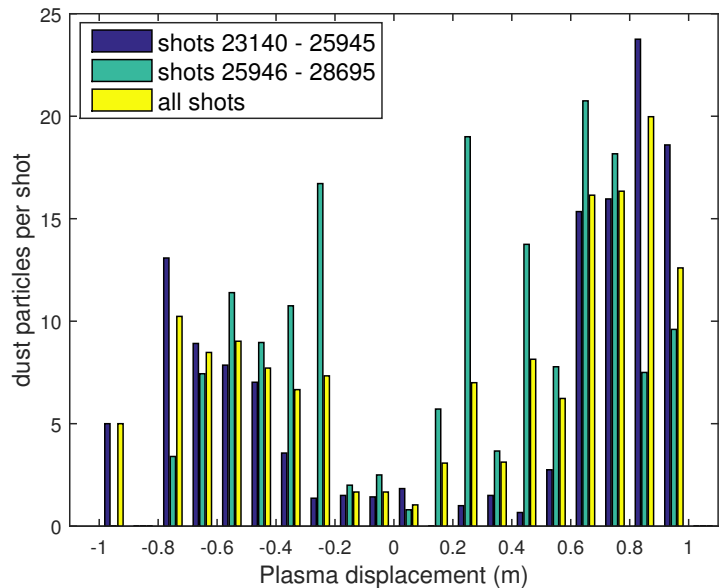

FIG. 9. Number of dust particles detected in disruptive discharges with vertical displacements as a function of the amplitude of the vertical displacement. Negative values of displacement correspond to downward VDEs and positive values to upward VDEs.

ELMs, but only seldom camera observations have been reported without any statistical assessment.

In order to investigate this issue in AUG, 69 nondisruptive discharges with significant unmitigated type-I ELM activity have been analyzed and compared to 983 non-disruptive discharges without type-I ELM activity (771 H-modes with types-II/III ELMs of much lower intensity and 212 L-modes) and to 479 disruptive discharges. In order to reduce the discrepancies resulting from various movie durations, the analysis presented in figure 10 focuses on the average number of dust particles detected per second for each shot. Please note that the average is computed over the total movie duration, which tends to soften the impact of transient events. This figure demonstrates that the probability to observe large dust rates is larger in the case of strong type-I ELM activity, with an average dust rate of $1.1 \mathrm{~s}^{-1}$, than in H-modes with type-II or type-III ELMs, where the average dust rate is $0.7 \mathrm{~s}^{-1}$. This denotes a rather mod- erate positive effect of type-I ELMs on dust production or remobilization. In both cases, dust observation rates are significantly larger than in L-mode discharges, where $N_{\text {dust }}=0.2 \mathrm{~s}^{-1}$, but significantly less than in disruptive discharges where an average value $N_{d u s t}=5.4 \mathrm{~s}^{-1}$ is found.

However, significant discrepancies can be observed according to the camera view. Indeed, the analysis of videos realized in sector 1 with the camera directed towards the divertor shows slightly opposite tendency, with $N_{\text {dust }}=0.35 \mathrm{~s}^{-1}$ in the case of strong type-I ELM activity and $N_{\text {dust }}=0.45 \mathrm{~s}^{-1}$ in the case of H-mode without type-I ELM activity. When the camera is imaging the upper part of the vessel, from sector 13 to sector 8 , it is found that $N_{\text {dust }}=1.55 \mathrm{~s}^{-1}$ in the case of strong type-I ELM activity and $N_{\text {dust }}=0.85 \mathrm{~s}^{-1}$ in the case of H-mode without type-I ELM activity. During type-I ELMs, the thermal radiation on the inner divertor can be so high that the camera sensor gets saturated for a few frames during and right after the interaction of filaments with the divertor. This effect can be seen in Fig. 1] For this reason, it is quite often impossible to detect any dust particle in the inner divertor region during such events and dust rates determined in the top-view configuration are likely to be underestimated. On the contrary, when camera is imaging the upper part of the vessel, frames are never saturated and variations in dust rates determined with TRACE do not suffer the same uncertainties. Therefore, the noticeable increase in dust rates observed in type-I ELMy H-modes in the upper part of the vessel denotes a fundamental trend, which illustrates the energy deposition on PFCs which are not designed to withstand high heat loads.

A more refined analysis of selected movies comparing the temporal evolution of significant dust releases with the one of type-I ELMs is presented in Figures 11(a-d). Type-I ELMs are easily evidenced by periodic bursts of the light intensity captured by fast cameras, resulting in fluctuations around $50-120 \mathrm{~Hz}$ in the power spectrum of light intensity. Apart from Fig 11(a) which suggests a possible correlation between dust release and a sudden increase in type-I ELM activity for a very short period of time $\left(\Delta N_{\text {dust }}=15\right.$ in between $\mathrm{t}=2.2$ and $\left.\mathrm{t}=2.5 \mathrm{~s}\right)$ and 
slow but regular increase of dust release during about $2 \mathrm{~s}$ of type-I ELM activity in Fig.11(b), most significant dust releases do not correspond to obvious type-I ELM activity. These observations demonstrate that type-I ELMs have an impact on the release of micron-sized dust in AUG, but this impact is not systematic and rather moderate, particularly in comparison to VDEs and disruptions.

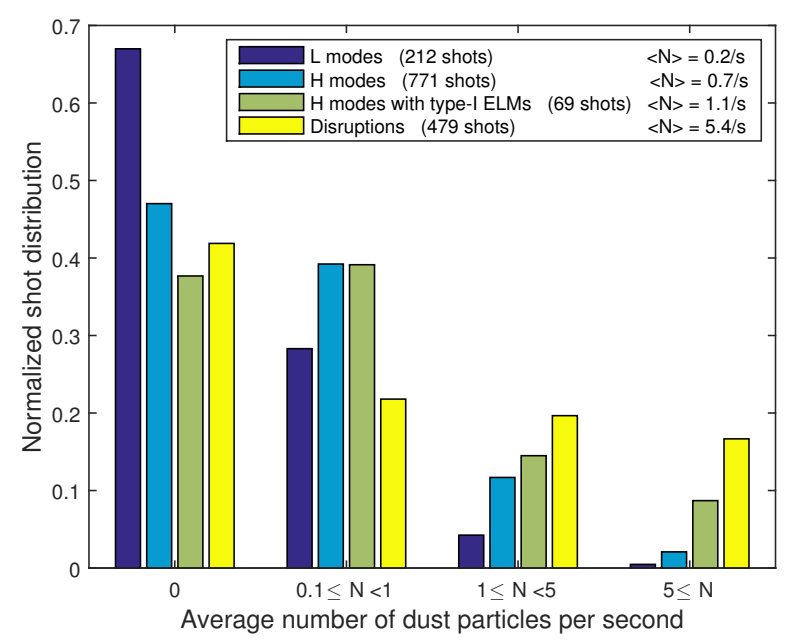

FIG. 10. Normalized distribution of shots as a function of average dust rates, per second, in non-disruptive $\mathrm{L}$ modes (dark blue), in non disruptive $\mathrm{H}$ modes without type-I ELM activity (light blue), in non-disruptive discharges with significant type-I ELMs activity (green) and in disruptive discharges (yellow).

\section{B. Influence of heating scenario}

It is well known that the coupling between ICRH power and the plasma leads to enhanced erosion of the antennas and of their limiter 32, 33. It is questionable whether this erosion results in the formation of dust observable with fast camera and of even bigger interest to assess the impact of various heating scenarii on the rates of dust observed by fast cameras. As shown in Figure 1, there is always at least one ICRH antenna in the camera field of view and two of them in most cases. Figure 12 presents the distribution of shots as a function of representative dust levels, according to the total heating power, integrated by steps of $1 \mathrm{MW}$. A total of 989 non-disruptive discharges have been considered. This figure shows that as the total heating power is increased from 2 to $14 \mathrm{MW}$, there is an increasing probability to observe significant dust events. Then, as the total heating power increases from $14 \mathrm{MW}$ to $20 \mathrm{MW}$, the probability of observing major dust events decreases, but it should be noted that the heating power is larger than $14 \mathrm{MW}$ in only 8 shots, i.e. $0.8 \%$ of the shots analyzed. In order to draw any firmer
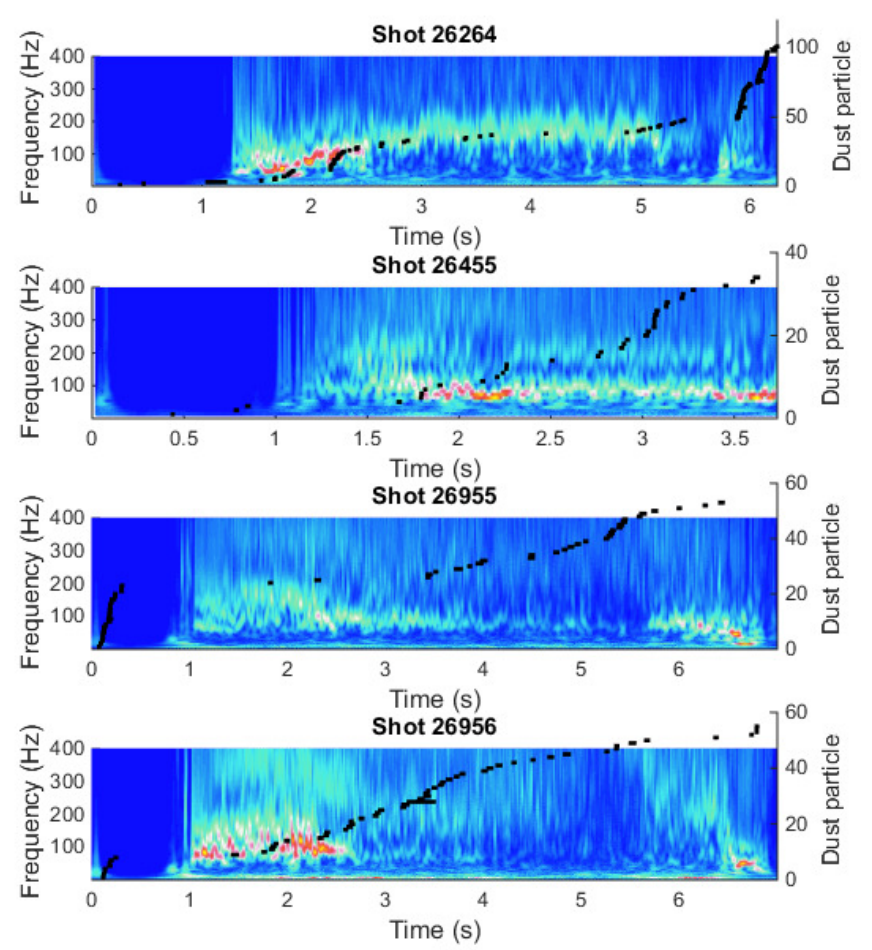

FIG. 11. Temporal evolution of the number of dust events (black dashs) superimposed to the Morlet wavelet power spectrum of light fluctuations measured by fast cameras in four elmy discharges exhibiting significant amount of dust events. The type-I ELM activity is evidenced by light fluctuations in the frequency range $50-120 \mathrm{~Hz}$ (in pink color). Shot 26264 is with camera imaging from sector 13 to 8 and the three other shots with camera imaging from sector 13 to 1 (see Fig. 1 ).

conclusion on the trend for large heating power, more camera data will be necessary.

To further investigate the influence of the heating scenario, Figure 13 details the dust rates per MW of heating power for all the possible combinations of heatings sources, in non-disruptive shots. If one excepts scenarios based on ECRH combined with ICRH only, due to low statistics, one remarks that the scenario based on the use of ECRH alone is the most likely to produce observable dust, with a dust rate of $0.35 / \mathrm{s} / \mathrm{MW}$. Then comes the scenario based on the use of NBI alone with a dust rate of $0.2 / \mathrm{s} / \mathrm{MW}$. Interestingly, the combination of ECRH and NBI has a beneficial effect since the dust rate goes down to $0.1 / \mathrm{s} / \mathrm{MW}$. One can also remark that the lowest rates are observed when ICRH is used, alone or in combination with other heating sources, which is rather unexpected. It is important to remember that this survey, which mixes up observations made with various camera views, depicts rather global effects. Given the variety of camera views and the locations of heating systems, as 
depicted in Figure 1, the antennas under operation in selected shots might be out of the camera field of view. A more detailed investigation of the influence of heating scenarios on dust rates is a very demanding task. More advanced conclusions will be presented in a separate paper.

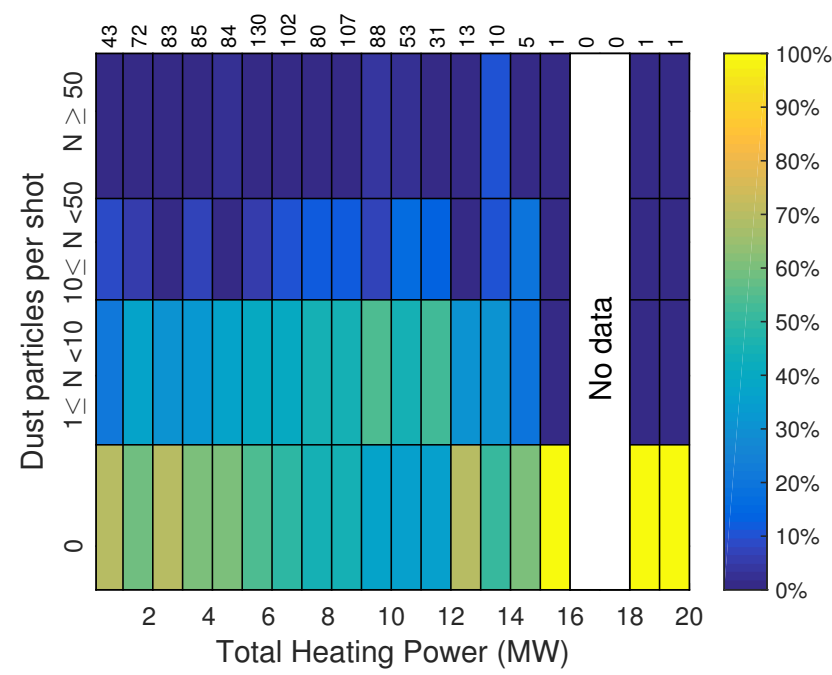

FIG. 12. Distribution of the number of dust particle per shot as a function of the total heating power in non-disruptive shots. The color scale represents the ratio of shots with given dust rates in given ranges of heating power. Numbers at the top of the graph give the number of data sets for each range of heating power.

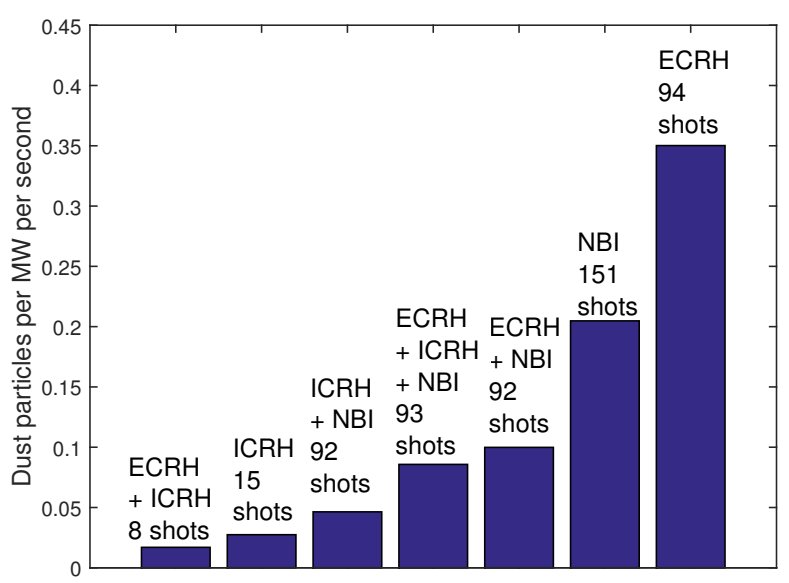

FIG. 13. Number of dust particles per second per MW of heating power for various combinations of heating sources. Only non-disruptive shot are considered.

\section{Influence of major vents}

In a previous study based on the analysis of standard cameras data, Hong et al. concluded that dust releases where significantly larger for several hundreds of discharges after vents in AUG and Tore Supra 4 . Rudakov et al. also report that dust observation rates with both standard rate cameras and fast cameras are significantly higher in DIII-D in the first shots after entry vents and are reduced to the normal operation rates after a few tens of shots 31. In AUG, first tens of shots after venting primarily aim at conditionning the walls and calibrating diagnostics. These discharges are only seldomly filmed with fast cameras, making it impossible to assess any short-term effect of vents with TRACE. Figure 14 depicts the number of dust particles per second detected with TRACE in seven different campaigns. Except for the third campaign, where fast camera data are available shortly after the start of the campaign $\left(15^{\text {th }}\right.$ shot -24205-), at least thirty shots took place before fast camera data are available in the other campaigns. It can be seen that the amount of dust observed in the beginning of the third campaign is significantly larger than in the rest of the campaign (about 20 times the average value). After repeated breakdown issues, tiles were cleaned after shot 24190 in order to operate with pure W surfaces. Dust rates then significantly decay within a few shots. It is interesting to note that the average dust rate is the lowest in this third campaign, which was conducted without any boronization. However, the limitations of the absence of regular wall conditionning during campaign $\sharp 24190$ - $\sharp 24990$ are evidenced if the beginning of the following campaign, which started without any vent, and which has one of the largest average dust rate. It is worth noting that the camera location was not changed during the third campaign and the first part of the fourth campaign, making it possible to have reliable statistics. Apart from the third campaign (shots 24190-25018), only the last analyzed campaign presents dust rate obviously larger in the first tens of shots after restart. All in all, regardless of the discharge conditions, the automated analysis of fast camera data may suggest short-term impact of vessel venting but does not reveal any long-term trend in AUG. This observation is further confirmed by the examination of 105 standard H-mode discharges, carried out at the beginning of each operation day to check AUG main systems, all with the same discharge parameters provided in Table II The dust rates observed in these discharges, depicted in Figure15, do not decay as a function of plasma operation time since previous vent. On addition, these rates do not seem to be influenced by boronizations, but rather seem to depend on the discharge conditions encountered in the previous shots.

Thus, our analysis suggests that vents do not have noticeable long-term effects on dust creation or mobilization, contrary to the tendency observed by S.H. Hong et al. [4] in the 2007 campaign ( $\sharp 22000$ series). This cam- 
paign, which started without wall boronization [34, was the first one after the completion of the all-W divertor. The installation of fast cameras on AUG took place after the 2007 campaign, making it impossible to analyze this campaign with TRACE, but it has to be noted that an extensive cleaning of the tiles was carried out in order to remove boron layers after shot 23188 , i.e. right before the first campaign analyzed in our study. Therefore, the differences between the long-term decay in dust observation rates observed in the 2007 campaign and our results are likely to be due to different wall conditionning, our analysis demonstrating that with appropriate wall conditionning, reactor maintenance does not lead to any significant production of dust.

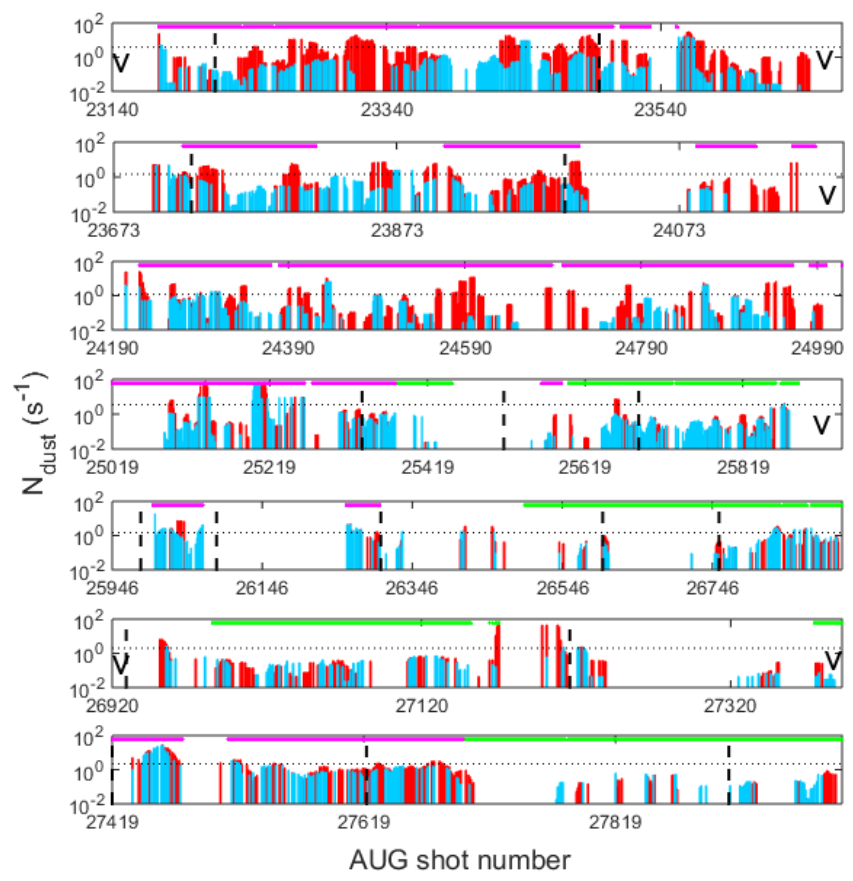

FIG. 14. Number of dust particles detected per second in AUG in 7 consecutive experimental campaigns. Disruptive shots are depicted in red and non-disruptive shots in blue. The dashed horizontal line shows the average value of $N_{d u s t}$ over each campaign. Shots with the camera imaging from sector 13 to 8 are highlited by magenta horizontal line and shots with the camera viewing the divertor from the top of sector 1 by horizontal green lines. The ' $\mathrm{V}$ ' letter is used to indicate major vents, which took place after shots 23138, 23654, 24189, 25890, 26919 and 27392. Finally, vertical dashed lines indicate boronizations.

\section{Influence of vessel vibrations}

It is questionable whether the remobilization of dust deposited onto the PFCs can be achieved or facilitated by vibrations of the latter. Despite it is not possible to measure directly PFCs vibrations during plasma operation,

\begin{tabular}{|c|c|}
\hline Parameters & Values \\
\hline Plasma current $\left(I_{p}\right)$ & $1 \mathrm{MA}$ \\
\hline Safety factor $\left(q_{95}\right)$ & 4.6 \\
\hline Auxiliary heating & $\sim 5 \mathrm{MW}$ \\
\hline Electron density $\left(n_{e}\right)$ & $5 \mathrm{e} 19 \mathrm{~m}^{-3}$ \\
\hline Toroidal magnetic field $\left(B_{T}\right)$ & $-2.47 \mathrm{~T}$ \\
\hline
\end{tabular}

TABLE II. Main discharge parameters used for AUG standard H-Mode discharges.

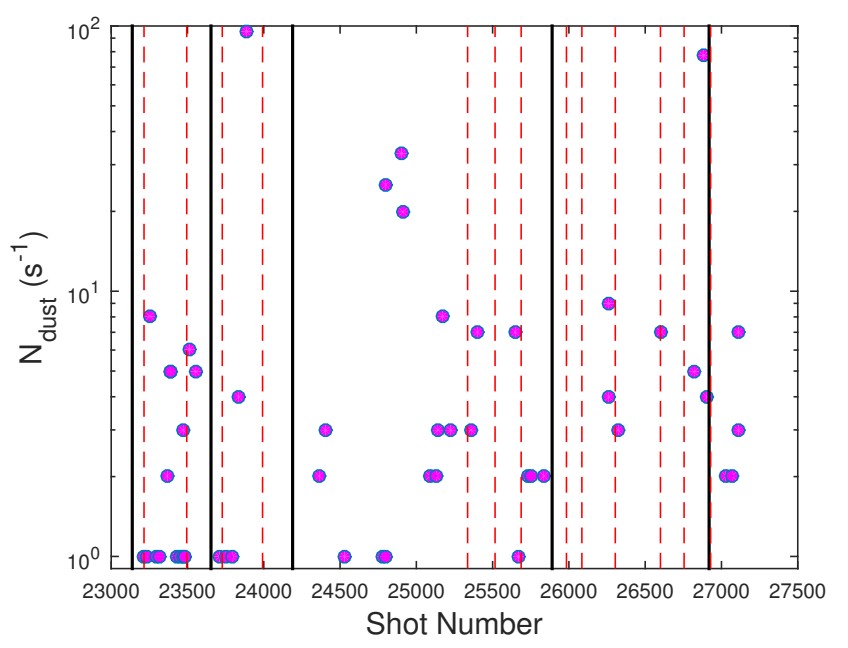

FIG. 15. Number of dust particles detected per second in AUG in standard H-Mode discharges in 6 consecutive experimental campaigns. The vertical black lines indicate the five major vents which took place during these campaigns, while red dashed lines indicate boronizations.

the dynamic deformations of some in-vessel components can be studied. AUG includes a Passive Stabilisation Loop (PSL) made of 2 rings equipped with a set of optical strain sensors. These sensors are connected to a data acquisition system with a sampling rate of $1 \mathrm{kHz}$, making it possible to resolve the potentially dangerous bending eigenmodes of the PSL and to measure vibrations resulting, for instance, of impulse forces during disruptions or PSL vibrations due to the actuation of the B-coils [35, which are typically in the range of $10-120 \mathrm{~Hz}$.

Strain measurements have been carried out for 68 shots covering all kinds of discharge conditions in between shots \#26023 and \#26264, but the Phantom v7.3 fast camera on which our dust analysis is based was used for only 17 of these shots. In order to obtain more complete results, we also include the results obtained with the analysis of 2 other fast cameras which have been used in the same series. 46 shots have been filmed with a Phantom v7.1 camera and 65 shots with a Photron SA1.1 camera, making it possible to analyze all the 68 shots, with two cameras for most of them. The 3 cameras where imaging comparable volume of AUG between sectors 8 to 13 , but with different lines of sight. Both Phantom cameras sensors have the same technical per- 
formances, yielding detection of similar amount of dust events, whereas the analysis of Photron camera data result in the detection of about 20 times more dust trajectories. For this reason, Phantom camera results have been mixed up whereas Photron camera results are presented separately. Figure 16 depicts the number of dust fly-bys detected by TRACE as a function of the maximum strain amplitudes measured in these 68 shots. The maximum amplitude $\left|\epsilon_{\max }\right|$ corresponds to the maximum peak amplitude measured by sensors located in sectors 8 to 13, i.e. sectors which are in the camera field of view. It can be seen that maximum strain amplitudes are measured during disruptive shots, as already shown in Ref. 36] but regardless of the discharge conditions, there is no correlation between $\left|\epsilon_{\max }\right|$ and the amount of dust observed with fast cameras. Vibration of the vessel are therefore unlikely to play any significant role in dust remobilization.

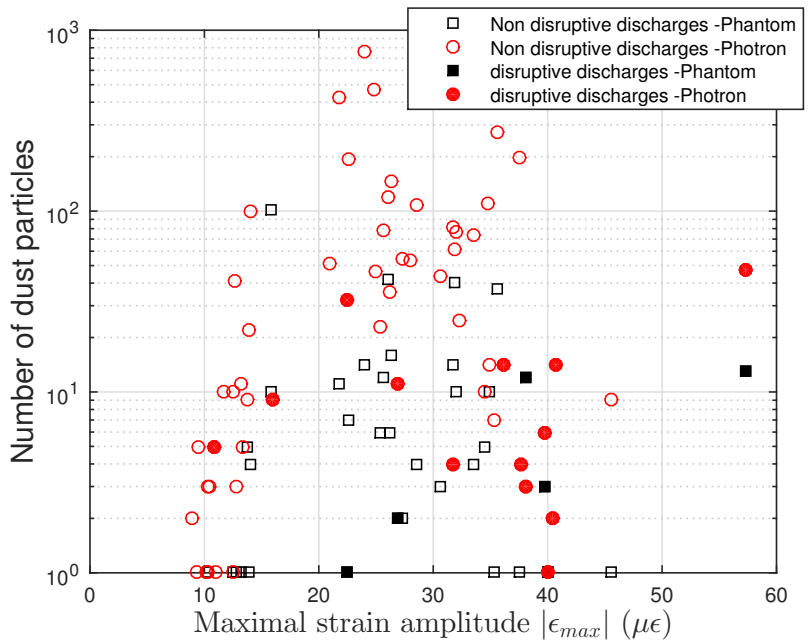

FIG. 16. Influence of vessel vibrations on dust remobilization: number of dust events as a function of the maximum strain amplitude measured on the PSL. Black square symbols represent results obtained with Phantom cameras while red circles depict results obtained with Photron camera. Hollow symbols are used for non-disruptive discharges and filled symbols for disruptions.

\section{DISCUSSION}

It is very challenging and actually impossible to suppress all the possible biases in such a statistical survey. Strictly speaking, good statistics would require analyzing data sets of discharges run with the same plasma conditions, PFCs and diagnostic setup, whereas tokamak discharges are never totally identical, the surface of PFCs is constantly changing and camera setup has been modified several times in the course of the analyzed campaigns. A lot of care was taken to filter out as much as possible bi- ases resulting from one or the other of these limitations. From a technical point of view, there are several factors which impact the effectiveness of dust detection. In addition to the technical aspects presented in section II-A (parameters influencing dust light emission, variability of the camera setups...) and in section II-B (counting of the only particles tracked at least 20 frames with a total displacement of at least 5 pixels, satisfying the maximal displacement criterion), one has to keep in mind that dust cannot be detected in saturated portions of the images and that camera ordinary covers only about $1 / 6$ of the volume of the plasma chamber. Only the largest dust particle which are not originating from a surface in the camera field of view have a chance to be detected $(100 \mu \mathrm{m}$ dust particles cover a maximal distance of about one meter in standard plasma conditions [18). Therefore, the absence of dust detection does not mean that plasma is dust-free.

Dust rates are particularly susceptible to be underestimated during transient events. Indeed, the latter tend to produce partially saturated images when the energy is deposited on PFCs that are in the field of view of the optical system, as discussed in section IV.A, and when the energy is deposited elsewhere dust particles which may be produced are unlikely to survive long enough to be detected. Also, the average dust rate per second, used in sections IV-A and IV-B in order to mitigate biases due to various movie durations, tends to lower the impact of transient events since the average is calculated by considering the total duration of the video.

This paper primarily aims at comparing the influence of various discharge conditions on dust rates and conclusions are mostly qualitative. Fast cameras make it possible to take benefit of the high temporal resolution to investigate the influence of transient events in details, as partially shown in Fig,11, thus enabling finest analysis. Such an investigation, exploiting also the spatial information, is underway and will certainly enable to draw more advanced conclusions on the impact of transient events and on the influence of the heating scenario on dust generation and remobilization.

\section{SUMMARY AND CONCLUSION}

A statistical survey of 2425 fast camera movies recorded from 2008 to 2012 in the ASDEX Upgrade tokamak has been carried out with the TRACE algorithm, with the aim to assess dust observation rates under various discharge conditions. These rates are found to be lower than in tokamaks with carbon PFCs. An average rate of 1.35 event per second is found and $52 \%$ of shots appear as free of visible moving dust in the camera field of view.

The largest dust observation rates are encountered in disruptive discharges, particularly those preceded by upward plasma vertical displacements. Dust rates increase with the amplitude of the displacement and with the dis- 
ruption energy, up to about $10^{5} \mathrm{~J}$ where a maximum is reached, which might result from dust melting on the PFCs or from insufficient data set at larger energies. Type-I ELMs are also found to increase dust rates by a factor of about 1.6. Vessel opening only impacts first tens of shots after restart and does not have any longterm effect. The almost constant dust rate during a campaign denotes the absence of building up of thick deposits on the W PFCs. The influence of heating scenario has also been investigated, pointing out enhanced dust rates in scenarios based on ECRH or NBI used alone, while ICRH might play an unexpected mitigative role. Finally, no correlation was found between the amplitude of vessel vibrations and dust observation rates, demonstrating that such vibrations have a negligible effect, if any, on dust remobilization.

The present work does not make it possible to assess whether dust is produced or only remobilized under given discharge conditions. It is questionable whether the increase in dust observation rates under transient heat fluxes is due to dust remobilization by heat loads, since the latter can also melt metallic dust and therefore increase dust adhesion to the PFCs, or to dust generation by arcs, since unipolar arcs are likely to be promoted by ELMs and disruptions. Dedicated experiments are required to draw firmer conclusions. For that purpose, the remobilization of pre-deposited tungsten dust under the influence of type-I ELMs is being investigated in AUG and complementary investigations on unipolar arcs will be carried out.

The detailed analysis of fast camera data recorded in AUG is still ongoing. The analysis of dust formation areas is now being carried out for various camera fields of view, which requires further recalibration work to adjust the camera lines of sight and has to take into account the evolutions in PFCs geometry and composition. Spatial results will enable a more detailed investigation of the influence of the heating scenario, in particular regarding the use of ICRH. In parallel to the $2 \mathrm{D}$ measurements analyzed in this paper, stereoscopic fast camera data have been carried out in several tens of shots, making it possible to reconstruct dust $3 \mathrm{D}$ trajectories. A study presenting the main characteristics of dust transport in AUG, aiming at better determining areas of dust formation, remobilization and deposition, will be presented in a separate paper. Such a work is crucial to help anticipating possible dust accumulation sites and to better estimate dust remobilization rates under normal and offnormal conditions as well as during dedicated cleaning discharges.

\section{ACKNOWLEDGEMENTS}

This work, supported by the European Community under the contract of Association between EURATOM, CEA, and the French Research Federation for fusion studies, was carried out within the framework of the EFDA (European Fusion Development Agreement) Task Force on Plasma Wall Interactions. The views and opinions expressed herein do not necessarily reflect those of the European Commission. Financial support was received from the French National Research Agency through Contract ANR-08-JCJC-0068-01 and A.S acknowledge the support of the Erasmus Mundus International Doctoral College in Fusion Science and Engineering (FUSION-DC).
[1] J. Winter, Plasma Phys. Control. Fusion 46, B583 (2004).

[2] V. Tsytovich and J. Winter, Physics Uspekhi 41, 815 (1998).

[3] M. Shoji, H. Kasahara, H. Tanaka, T. Murase, M. Tokitani, S. Morita, M. Goto, T. Oishi, T. Mutoh, E. D. L. Cal, C. Hidalgo, and the LHD Experiment Group, Plasma and Fusion Research 11, 2402056 (2016).

[4] S. Hong, C. Grisolia, V. Rohde, P. Monier-Garbet, T. S. Team, and A. U. Team, Nucl. Fusion 50, 035002 (2010).

[5] B. Pégourié, C. Brosset, E. Tsitrone, A. Beauté, S. Brémond, J. Bucalossi, S. Carpentier, Y. Corre, E. Delchambre, C. Desgranges, P. Devynck, D. Douai, G. Dunand, A. Ekedahl, A. Escarguel, E. Gauthier, J. Gunn, P. Hertout, S.-H. Hong, F. Kazarian, M. Kočan, F. Linez, Y. Marandet, A. Martinez, M. Mayer, O. Meyer, P. Monier-Garbet, P. Moreau, P. Oddon, J.-Y. Pascal, F. Rimini, J. Roth, F. Saint-Laurent, F. Samaille, S. Vartanian, C. Arnas, E. Aréou, C. Gil, J. Lasalle, L. Manenc, C. Martin, M. Richou, P. Roubin, and R. Sabot, J. Nucl. Mater. 390-391, 550 (2009).
[6] B. Bray, W. West, and R. Rudakov, J. Nucl. Mater. 390-391, 96 (2009).

[7] S. Ratynskaia, C. Castaldo, E. Giovannozzi, D. Rudakov, G. Morfill, M. Horanyi, J. Yu, and G. Maddaluno, Plasma Phys. Control. Fusion 50, 124056 (2008).

[8] C. Skinner, B. Rais, A. Roquemore, H. Kugel, R. Marsala, and T. Provost, Rev. Sci. Instrum. 81, 10E102 (2010).

[9] H. Roche, A. Barbuti, J. Bucalossi, L. Ducobu, C. Grisolia, T. Loarer, B. Pégourié, S. Rosanvallon, P. Spuig, C. Skinner, S. Vartanian, and B. Vincent, Physica Scripta T145, 014022 (2011).

[10] K. Kim, S. Hong, Y. Nam, J. Jung, W. Kim, and the KSTAR Team, Journal of Nuclear Materials 438, S719 (2013).

[11] J. Yu, D. Rudakov, A. Pigarov, R. Smirnov, N. Brooks, S. Muller, and W. West, J. Nucl. Mater. 390-391, 216 (2009).

[12] J. Sharpe, V. Rohde, the ASDEX Upgrade Experiment Team, A. Sagara, H. Suzuki, A. Komori, O. Motojima, and the LHD Experimental Group, J. Nucl. Mater. 313-316, 455 (2003). 
[13] N. Endstrasser, V. Rohde, M. Balden, P. Humrickhouse, U. von Toussaint, B. Braams, H. Chung, R. Neu, and the ASDEX Upgrade Team, Phys. Scr. T145, 014021 (2011).

[14] M. Balden, N. Endstrasser, P. Humrickhouse, V. Rohde, M. Rasinski, S. Lindig, R. Neu, and the ASDEX Upgrade Team, Nucl. Fusion 54, 073010 (2014).

[15] Y. Zayachuk, F. Brochard, S. Bardin, J.-L. Briançon, R. Hugon, and J. Bougdira, arXiv:1010.3432v1 (2010)

[16] N. Endstrasser, F. Brochard, V. Rohde, M. Balden, T. Lunt, S. Bardin, J. Briançon, R. Neu, and the ASDEX Upgrade Team, Journal of Nuclear Materials 415, S1085 (2011).

[17] E. Marenkov and S. Krasheninnikov, Physics of Plasmas 21, 123701 (2014).

[18] Z. Yang, K. Krieger, T. Lunt, F. Brochard, J.-L. Briançon, R. Neu, R. Dux, A. Janzer, S. Potzel, T. Puetterich, and the ASDEX Upgrade Team, J. Nucl. Mater. 438, S846 (2013).

[19] R. Smirnov, S. Krasheninnikov, J. Yu, A. Y. Pigarov, M. Rosenberg, and J. Terry, Plasma Phys. Control. Fusion 51, 055017 (2009).

[20] D. Rudakov, J. Yu, J. Boedo, E. Hollmann, S. Krasheninnikov, R. Moyer, S. Muller, A. Pigarov, M. Rosenberg, R. Smirnov, W. West, R. Boivin, B. Bray, N. Brooks, A. Hyatt, C. Wong, A. Roquemore, C. Skinner, W. Solomon, S. Ratynskaia, M. Fenstermacher, M. Groth, C. Lasnier, A. McLean, and P. Stangeby, Rev. Sci. Instrum. 79, 10F303 (2008).

[21] D. Rudakov, W. West, C. Wong, N. Brooks, T. Evans, M. Fenstermacher, M. Groth, S. Krasheninnikov, C. Lasnier, A. McLean, A. Pigarov, W. Solomon, G. Antar, J. Boedo, R. Doerner, E. Hollmann, A. Hyatt, R. Moyer, and J. Watkins, Journal of Nuclear Materials 363-365, 227 (2007).

[22] A. Shalpegin, L. Vignitchouk, I. Erofeev, F. Brochard, A. Litnovsky, S. Bozhenkov, I. Bykov, N. den Harder, and G. Sergienko, Plasma Phys. Control. Fusion 57, 125017 (2015).

[23] A. Shalpegin, F. Brochard, S. Ratynskaia, P. Tolias, M. D. Angeli, L. Vignitchouk, I. Bykov, S. Bardin, K. Bystrov, T. Morgan, and G. D. Temmerman, Nucl. Fusion 55, 112001 (2015).

[24] S. Dap, D. Lacroix, R. Hugon, L. de Poucques, J.-L. Briançon, and J. Bougdira, Phys. Rev. Lett. 109, 245002 (2012).

[25] A. Melhem, G. Henrion, T. Czerwiec, J.-L. Briançon, T. Duchanoy, F. Brochard, and T. Belmonte, Surf. Coat.
Technol. 205, S133 (2011).

[26] S. Krasheninnikov, A. Y. Pigarov, R. Smirnov, M. Rosenberg, Y. Tanaka, D. Benson, T. Soboleva, T. Rognlien, D. Mendis, B. Bray, D. Rudakov, J. Yu, W. West, A. Roquemore, C. Skinner, J. Terry, B. Lipschultz, A. Bader, R. Granetz, C. Pitcher, N. Ohno, S. Takamura, S. Masuzaki, N. Ashikawa, M. Shiratani, M. Tokitani, R. Kumazawa, N. Asakura, T. Nakano, A. Litnovsky, R. Maqueda, and the LHD Experimental Group, Plasma Phys. Control. Fusion 50, 124054 (2008).

[27] Y. V. Martynenko and M. Y. Nagel, Plasma Physics Reports 38, 290 (2012).

[28] P. Tolias, S. Ratynskaia, M. D. Angeli, G. D. Temmerman, D. Ripamonti, G. Riva, I. Bykov, A. Shalpegin, L. Vignitchouk, F. Brochard, K. Bystrov, S. Bardin, and A. Litnovsky, Plasma Phys. Control. Fusion 58, 025009 (2016).

[29] J. Coenen, V. Philipps, S. Brezinsek, B. Bazylev, A. Kreter, T. Hirai, M. Laengner, T. Tanabe, Y. Ueda, U. Samm, and the TEXTOR team, Nucl. Fusion 51, 083008 (2011).

[30] G. Pautasso, C. Fuchs, O. Gruber, C. Maggi, M. Maraschek, T. Pütterich, V. Rohde, C. Wittmann, E. W. Cierpka, M. Beck, and the ASDEX Upgrade Team, Nucl. Fusion 47, 900 (2007).

[31] D. Rudakov, A. Litnovsky, W. West, J. Yu, J. Boedo, B. Gray, S. Brezinsek, N. Brooks, M. Fenstermacher, M. Groth, E. Hollmann, A. Huber, A. Hyatt, S. Krasheninnikov, C. Lasnier, A. McLean, R. Moyer, A. Pigarov, V. Philipps, A. Pospieszczyk, R. Smirnov, J. Sharpe, W. Solomon, J. Watkins, and C. Wong, Nuclear Fusion 49, 085022 (2009).

[32] J. M. Noterdaeme and G. van Oost, Plasma Phys. Control. Fusion 35, 1481 (1993).

[33] S. Wukitch, B. Lipschultz, E. Marmar, Y. Lin, A. Parisot, M. Reinke, J. Rice, J. Terry, and the Alcator C Mod Team, Journal of Nuclear Materials 363-365, 491 (2007).

[34] R. Neu, M. Balden, V. Bobkov, R. Dux, O. Gruber, A. Herrmann, A. Kallenbach, and et al., Plasma Phys. Control. Fusion 49, B59 (2007).

[35] C. Vorpahl, W. Suttrop, M. Ebner, B. Streibl, H. Zohm, and the ASDEX Upgrade Team, Fusion Eng. Des. 88, 537 (2013).

[36] C. Vorpahl, Investigation of the deformation of in-vessel components of a nuclear fusion experiment using optical strain sensors, Ph.D. thesis, Karlsruher Institute für Technologie (2013). 